\title{
Dynamic Hsp83 RNA Localization during Drosophila Oogenesis and Embryogenesis
}

\author{
DALI DING, SUSAN M. PARKHURST, $†$ SUSAN R. HALSELL, AND HOWARD D. LIPSHITZ* \\ Division of Biology, 156-29, California Institute of Technology, Pasadena, California 91125
}

Received 14 December 1992/Returned for modification 20 January 1993/Accepted 18 March 1993

\begin{abstract}
Hsp83 is the Drosophila homolog of the mammalian $\mathrm{Hsp} 90$ family of regulatory molecular chaperones. We show that maternally synthesized $\mathrm{Hsp} 83$ transcripts are localized to the posterior pole of the early Drosophila embryo by a novel mechanism involving a combination of generalized RNA degradation and local protection at the posterior. This protection of $\mathrm{Hsp83}$ RNA occurs in wild-type embryos and embryos produced by females carrying the maternal efiect mutations nanos and pumilio, which eliminate components of the posterior polar plasm without disrupting polar granule integrity. In contrast, $H s p 83$ RNA is not protected at the posterior pole of embryos produced by females carrying maternal mutations that disrupt the posterior polar plasm and the polar granules - cappuccino, oskar, spire, staufen, tudor, valois, and vasa. Mislocalization of oskar RNA to the anterior pole, which has been shown to result in induction of germ cells at the anterior, leads to anterior protection of maternal $\mathrm{Hsp83}$ RNA. These results suggest that $\mathrm{Hsp83}$ RNA is a component of the posterior polar plasm that might be associated with polar granules. In addition, we show that zygotic expression of $\mathrm{Hsp} 83$ commences in the anterior third of the embryo at the syncytial blastoderm stage and is regulated by the anterior morphogen, bicoid. We consider the possible developmental significance of this complex control of $\mathrm{Hsp} 83$ transcript distribution.
\end{abstract}

Cytoplasmically localized determinants, in the form of localized maternal RNAs and proteins, play a key role in providing positional cues in the oocyte and early embryo of Drosophila melanogaster. To date, two RNAs localized to the anterior pole of both the oocyte and the early embryo have been described: bicoid RNA, which encodes the anterior determinant $(12,13,58,59)$, and RNA encoding a Drosophila adducin homolog (11). A number of maternally active genes encode posteriorly localized molecules. Proteins encoded by germ cell-less, oskar, vasa, and staufen and RNAs encoded by cyclin B, germ cell-less, nanos, orb, oskar, pumilio, and tudor are localized components of the posterior polar plasm, a posteriorly located, yolk-free cytoplasmic cap that is continuous with the cortical cytoplasm of the egg and early embryo $(14,15,18,22-24,28,30,35-37$, $43,45,54,57,63)$. Important components of this polar plasm are the polar granules-electron-dense, non-membranebound organelles-that reside within $4 \mu \mathrm{m}$ of the plasma membrane at the posterior tip of the oocyte and early embryo $(27,46-49)$. The polar granules are taken up into the pole cells-the Drosophila primordial germ cells of-as these cells bud off the posterior end of the embryo and have been postulated to play a key role in programming them to adopt germ line fates.

Many maternally transcribed genes with spatially restricted functions in the Drosophila embryo have been identified in genetic screens $(5,17,39,41,42,50,55,56)$. However, it has been estimated that only one-third of the transcription units of the Drosophila genome have been genetically defined (4). To isolate additional maternal molecules with spatially restricted functions, we carried out a differential screen for cDNAs representing RNAs that are localized to either the anterior or the posterior pole of the

\footnotetext{
* Corresponding author. Electronic mail address: LIPSHITZH @STARBASE1.CALTECH.EDU.

$\dagger$ Present address: Division of Basic Sciences M-616, Fred Hutchinson Cancer Research Center, Seattle, WA 98104.
}

Drosophila oocyte or early embryo (10). Apart from studying their possible developmental roles, we expected that identification of localized RNAs would be useful in the analysis of RNA localization mechanisms per se $(9,10)$. Here we report a detailed analysis of the spatial localization of one of the posterior-localized RNAs we identified in this screen. It is encoded by the Hsp83 gene, the sole Drosophila homolog of the mammalian $H s p 90$ gene family $(2,10,21)$.

It has been known for some time that Drosophila Hsp83 is not only heat inducible but also expressed at high levels during normal development $(33,65,66)$. Despite the extensive biochemical studies of the $H s p 90$ family of cytoplasmically active regulatory molecular chaperones in mammals, Saccharomyces cerevisiae, and D. melanogaster, little is known about their developmental regulation and functions (for a review, see reference 44). While mutational analyses of the Hsp82 genes have shown that Hsp82 is an essential protein in S. cerevisiae (3), no Drosophila Hsp83 mutations have been identified (64).

Here, we show that maternal Drosophila Hsp83 RNA is concentrated at the posterior pole of the early embryo via a novel RNA localization mechanism involving a combination of generalized degradation throughout the embryo and local protection of $H s p 83$ RNA at the posterior pole. Analyses of the distribution of Hsp83 RNA in embryos produced by mutants lacking polar granules, as well as in embryos that have a key polar plasm component-oskar-mislocalized anteriorly, reveal that $H s p 83$ RNA is a component of the posterior polar plasm. $H s p \delta 3$ RNA is present at high levels in the germ line cells throughout most of development, with the exception of two periods during oogenesis. In addition, $H s p 83$ is transcribed zygotically in the anterior third of the embryo commencing at the syncytial blastoderm stage. This anterior zygotic expression is missing in embryos produced by bicoid mutant mothers, suggesting that early zygotic Hsp83 transcription may be controlled by the bicoid homeodomain protein. 


\section{MATERIALS AND METHODS}

Differential cDNA screen for polarly localized RNAs. Our differential screen for cDNAs representing polarly localized RNAs is described in detail elsewhere (10). Briefly, RNA was purified from anterior or posterior poles cut off frozen embryos and was used in the construction of directionally cloned cDNA libraries in $\lambda E X L X$ vectors (53). The entire anterior and posterior libraries were converted from phage to plasmid libraries by Cre-loxP automatic plasmid subcloning (53), and plasmid DNA was purified from each of these libraries. Probe generated from this anterior or posterior DNA was used to differentially screen $10^{5}$ plaques from a 0 - to 1-h whole embryo library constructed in $\lambda \mathrm{gt} 10$; rescreening of the clones on Southern blots and sorting into cross-hybridization classes followed. From this screen, we obtained 12 members of the SHTZ68 class of posterior clones (a frequency of $10^{-4}$ ). These were hybridized in situ to whole-mount early embryos (see below) to confirm that they encoded a posterior-localized RNA. Sequencing of these cDNAs followed by a sequence similarity search with FASTA as implemented in the Genetics Computer Group Sequence Analysis Package run at the Caltech Biology Division Sequence Analysis Facility revealed that the SHTZ68 cDNAs encode Hsp83 $(2,21)$. The Drosophila Hsp83 gene was cloned more than 13 years ago (26), and both its heat-inducible and its developmentally regulated expression have been studied in some detail $(33,65,66)$. However, it was the recloning of $H s p 83$ as the SHTZ68 class of cDNAs that uncovered both the posterior localization of maternal $H s p 83$ RNA and the anterior-restricted zygotic expression of $H s p 83$.

In situ hybridization to whole-mount ovaries and embryos. Whole-mount RNA tissue in situ hybridization was based on the method of Tautz and Pfeifle (61). Ovaries from adult females were dissected in phosphate-buffered saline (PBS), fixed for $25 \mathrm{~min}$ in $10 \%$ paraformaldehyde or formaldehyde-50 mM ethylene glycol-bis( $\beta$-aminoethyl ether)- $N, N$, $N^{\prime}, N^{\prime}$-tetraacetic acid (EGTA)-10\% dimethyl sulfoxide in PBS and washed several times in PBS plus $0.1 \%$ Tween 20. Ovaries were then rubbed gently between two frosted microscope slides in order to break apart the ovarioles and devitellinize the late egg chambers. Postfixation, proteinase $\mathbf{K}$ digestion, and refixation were as previously described (61). Embryos were fixed as previously described (61), with only minor modifications. Digoxigenin probes were labeled by random priming of DNA synthesis according to instructions from the manufacturer (Boehringer Mannheim) or by single-sided polymerase chain reaction amplification by a protocol provided by N. Patel (Carnegie Institution of Washington, Baltimore, Md.). Hybridization and detection were as previously described (61). Ovaries and embryos were mounted in JB4 plastic mountant for microscopy (Polysciences).

Temporal and quantitative analysis of $\mathrm{Hsp} 83$ localization in the early embryo. In order to analyze the time course of Hsp83 RNA localization, it was necessary to obtain precisely staged early embryos. Embryos were collected from well-fed wild-type females at 15 - to 20 -min intervals; thus, each collection contained embryos that differed only by a single nuclear cleavage cycle. The embryos were allowed to age at $25^{\circ} \mathrm{C}$ for different lengths of time prior to fixation in order to obtain material staged from fertilization through the completion of cellularization ( 0 to $2.5 \mathrm{~h}$ after egg deposition).

These embryos were then processed for in situ RNA hybridization with $H s p 83$ probes as outlined above. In order to quantify the intensity of the in situ hybridization signals, the color reaction was stopped early in order to understain the embryos, thus ensuring that the signal was not saturated. Images of whole-mount embryos were captured and digitized for computer analysis with a Dage-MTI CCD-72 Series solid-state camera (Dage-MTI, Inc., Michigan City, Ind.) and an Image Grabber NUBus digitizer board (Neotech, Ltd., Eastleigh, Hampshire, United Kingdom) installed in a Macintosh II computer. Initial processing of the image was carried out with Image Grabber software (version 2.01). Subsequent measurements and production of pseudocolor images representing the concentration distribution of $\mathrm{Hsp} 83$ transcripts were carried out with NIH Image public domain software (version 4.2; written by J. Ayers and G. Fletcher; available via anonymous file transfer protocol from sumexaim.stanford.edu). Measurements were then made of the average gray-scale values for pixels in 25 equisize areas along the midline of the anteroposterior axis (normalized for egg length). The equivalent values from a background image were subtracted. Five embryos from each stage were analyzed in this way, and the mean gray-scale values for each of the 25 areas were used to plot the relative intensity of $H s p 83$ in situ signal along the anteroposterior axis at two stages of early embryogenesis (see Fig. 2). This method is similar to that used by St. Johnston et al. (58) and Driever and Nüsslein-Volhard (13).

Fly strains. Mutant embryos were obtained from females homozygous for $\operatorname{osk}^{166}(39)$, capu $^{H K}(50), \operatorname{spir}^{R P}(50)$, nos $^{L 7}$ (41), pum ${ }^{680}$ (41), exu ${ }^{P J}(55)$, vas $^{P D}$ (55), stau ${ }^{H L}$ (55), vls ${ }^{R B}$ (55), tud ${ }^{W C 8}$ (55), and bcd ${ }^{E 1}$ (17). Mutants deficient for Hsp83 were provided by A. Wohlwill and J. J. Bonner (Indiana University) (64). Embryos lacking the Hsp83 gene were derived from crosses between parent flies with the following genotypes: $D f(3 L) H R 218 / D p(3 ; 3) t^{33} F 19^{R}, D f(3 L)$ $H R 298 / D p(3 ; 3) t^{33} F 19^{R}$, and $D f(3 L) H R 370 / D p(3 ; 3) t^{33} F 19^{R}(64)$. Homozygous $h b$ mutant embryos were derived from crosses between parent flies carrying $D f(3 R) h b^{P T X 15} p^{p} e / T M 3$ (40). Embryos with oskar RNA mislocalized to the anterior pole were obtained from females heterozygous for osk-bcd3'UTR as described previously (15). The $c 83 Z$. -880 strain of flies germ line transformed with a Hsp83-lacZ fusion gene was provided by $\mathrm{H}$. Xiao and J. Lis (Cornell University) (65).

\section{RESULTS}

Hsp83 RNA is expressed in a dynamic fashion during oogenesis. Drosophila oogenesis can be subdivided into distinct stages that occur in an ordered spatial and temporal array in the individual ovarioles that compose each ovary (31). At the anterior tip of the ovariole is the germarium, in which germ line stem cells divide asymmetrically to give rise to blast cells that will contribute the germ line components of the ovarian follicles. These undergo a series of four mitotic divisions with incomplete cytokinesis, forming an interconnected 16-cell germ line cyst. One of the cells forms the oocyte, and the remaining 15 form the nurse cells. The germarium is subdivided into three regions (31) (Fig. 1). The follicle grows in size and matures in the vitellarium, in a series of 14 stages (Fig. 1; stage 1 is equivalent to germarial stage 3) (31). Most of the synthesis of RNA and protein occurs in the nurse cells, and these molecules are then transported into the oocyte. In addition, yolk is transported to the oocyte in the hemolymph and is taken up by pinocytosis, contributing significantly to the increase in oocyte volume from stage 8 onwards.

$H s p 83$ RNA expression is absent in region 1 of the 


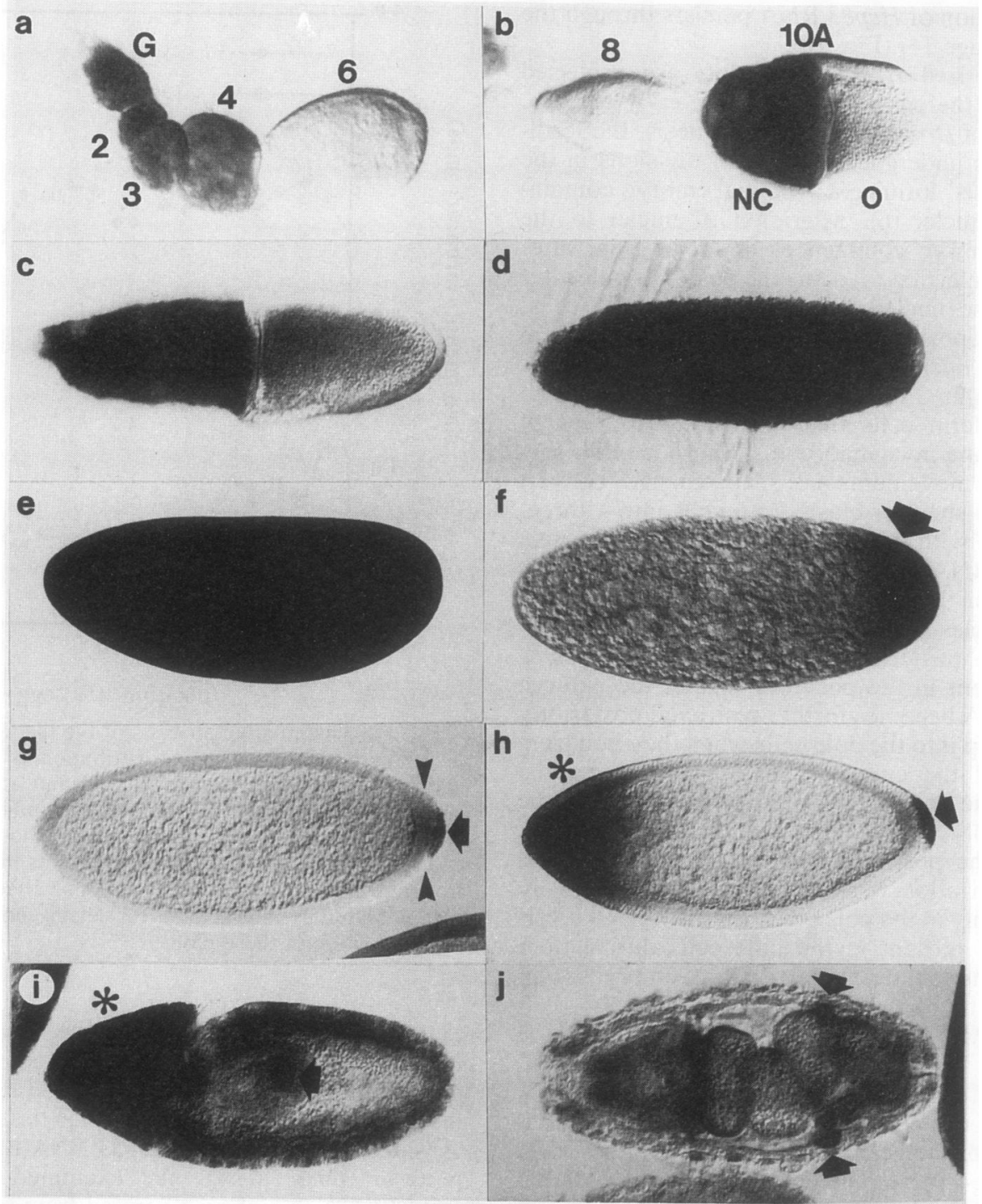

FIG. 1. Expression and localization of $H s p 83$ RNA during oogenesis and embryogenesis. In situ hybridization of the $H s p 83$ cDNA probe to ovarioles and embryos from wild-type females is shown. (a) During the early stages of oogenesis, $H s p 83$ expression is detected in all the cells in regions 2 and 3 of the germarium (G). In stage 1 through 5 egg chambers, Hsp83 is expressed in all 16 cells of the nurse cell-oocyte complex (shown are stage 1, which is equivalent to germarial stage 3, and stages 2, 3, and 4). No Hsp83 RNA is detected in stage 6 to 8 egg chambers (stage 6 is shown). (b) A stage 8 egg chamber (8) lacks detectable $H s p 83$ RNA, while a high level of $H s p 83$ expression is found again in the nurse cells beginning in stage 9. The high level of nurse cell (NC) expression can be seen in the stage 10A egg chamber shown. $\mathrm{O}$, oocyte. (c) In a stage 10B egg chamber, nurse cells contain high levels of $H s p 83$ RNA, which begins to be transported into the oocyte. (d) In stage 14 oocytes, Hsp83 is distributed uniformly at high levels. (e) In an early-cleavage-stage embryo (before nuclear cycle 6), maternal Hsp83 RNA is uniformly distributed and present at high levels. (f) In a nuclear cycle 7 embryo, maternal Hsp83 RNA is more concentrated at the posterior end (arrow), forming a decreasing gradient towards the anterior. (g) By the syncytial blastoderm stage, Hsp83 RNA is only detected in the pole cells (arrow) and a small region immediately beneath them (arrowheads). (h) At the cellular blastoderm stage, zygotic Hsp83 transcripts are present in the anterior third of the embryo (asterisk) in addition to the maternal RNA that is present in the pole cells (arrow). (i) During germ band extension, Hsp83 RNA is present at high levels in the pole cells (arrow), the head region (asterisk), and the neuroblasts. (j) In a fully developed embryo, Hsp83 RNA is present at high levels in the gonads (arrows). In all embryos except that shown in panel $\mathbf{j}$, anterior is to the left and dorsal is up. The view in panel $\mathbf{j}$ is from the dorsal side, again with anterior to the left.

germarium and is first detected in germarial regions 2 and 3 in all 16 cells of the germ line cyst (Fig. 1a). In stage 1 through 5 egg chambers, $H s p 83$ expression continues in all 16 cells of the nurse cell-oocyte complex (Fig. 1a). This RNA is rapidly degraded at the end of stage 5 , since $H s p 83$ RNA is present in stage 5 egg chambers but is undetectable in stage 6 egg chambers (Fig. 1a). It remains absent through stage 8 (Fig. 1b). High levels of $H s p 83$ RNA are then expressed again beginning at stage 9 , but only in the 15 nurse cells. This nurse cell expression persists through stages 10 and 11, and transport of Hsp83 RNA into the oocyte commences at stage 10B (Fig. 1c). Thus, Hsp83 RNA is absent from the oocyte for roughly $30 \mathrm{~h}$ spanning stages 6 through $10 \mathrm{~A}$ of oogenesis. By stage 12 , when nurse cells have completely emptied their contents into the oocyte, Hsp83 RNA is present at high levels throughout the oocyte. 
This high concentration of $H s p 83$ RNA persists through the end of oogenesis (stage 14) (Fig. 1d).

Maternally synthesized $\boldsymbol{H s p} 83$ transcripts are protected from degradation at the posterior pole of the early embryo. During the first $3 \mathrm{~h}$ of Drosophila embryogenesis, the fertilized zygotic nucleus undergoes a series of divisions in the absence of cytokinesis, forming a syncytial embryo containing roughly 6,000 nuclei (6). Migration of nuclei to the periphery of the embryo commences about an hour after fertilization, with nuclei first reaching the posterior pole after about $80 \mathrm{~min}$. These nuclei contribute to the pole cells, which bud from the posterior and are the primordial germ cells. The remaining nuclei continue to divide in the cortical cytoplasm and, roughly $3 \mathrm{~h}$ after fertilization, contribute to the somatic blastoderm cells that form by a process of coordinated membrane invagination (6). Subsequently, gastrulation-related cell movements and shape changes convert the two-dimensional sheet of blastoderm cells into a threedimensional embryo with distinct tissue layers and organs.

Maternal $H s p 83$ RNA is distributed throughout the early embryo from nuclear division cycles 1 through 5 (Fig. 1e). During cleavage cycles 6 to $8, H s p 83$ RNA is most highly concentrated at the posterior pole, forming a decreasing concentration gradient in the posterior half of the embryo (Fig. 1f). Hsp83 RNA becomes further restricted posteriorly before being taken up into the pole cells when they bud from the posterior tip of the embryo. Thus, by the syncytial blastoderm stage, the only detectable $H s p 83$ RNA is in the pole cells and a small region just beneath the pole cells (Fig. 1g). The RNA in the posterior somatic region disappears shortly after cellularization, leaving high levels of maternally synthesized RNA only in the pole cells (Fig. 1h). High levels of $H s p 83$ RNA are present in the pole cells during their migration (Fig. 1i) and in the gonads of mature embryos (Fig. $1 \mathrm{j})$, larvae, and adults $(65,66$; this study).

Localization of $H s p 83$ RNA to the posterior pole of the embryo is achieved by a combination of generalized turnover throughout the embryo and protection of Hsp83 RNA from degradation at the posterior pole (Fig. 2). An alternative-translocation of the generally distributed $H s p 83$ RNA to the posterior pole-is excluded by the fact that maternal $H s p 83$ transcripts are present at very high levels in newly fertilized eggs, and the concentration of $H s p 83$ RNA at the posterior pole remains constant rather than increasing (Fig. 2). In support of our quantitative analysis of $H s p 83$ RNA by tissue in situ hybridization (Fig. 2), Northern (RNA) blot analysis shows that there is less Hsp83 RNA in 2- to 4-h embryos than in 0 - to 2 -h embryos (data not shown). Embryos homozygous for deletions of $H s p 83$ still show posterior localization of $H s p 83$ RNA (see Fig. 5a), excluding the possibility that de novo zygotic expression of $H s p 83$ at the posterior of the embryo contributes significantly to the observed posteriorly localized $H s p 83$ RNA pool. Sequences required for posterior protection of the Hsp83 RNA map 3' to the translation initiation site, since a Hsp83-lacZ fusion RNA (see below) is no longer protected at the posterior pole of early embryos (see Fig. 6).

Zygotic transcription of $\mathrm{Hsp} 83$ is restricted to the anterior third of the embryo. At the late syncytial blastoderm stage, expression of $\mathbf{H s p 8 3}$ is detected in the anterior third of the embryo. This anterior expression continues through the cellular blastoderm stage (Fig. 1h), gastrulation (Fig. 1i), and most of embryogenesis. Anterior Hsp83 expression is eliminated from embryos homozygous for a deletion that removes the Hsp 83 locus, indicating that it results from de novo zygotic transcription (see Fig. 5a). During germ band

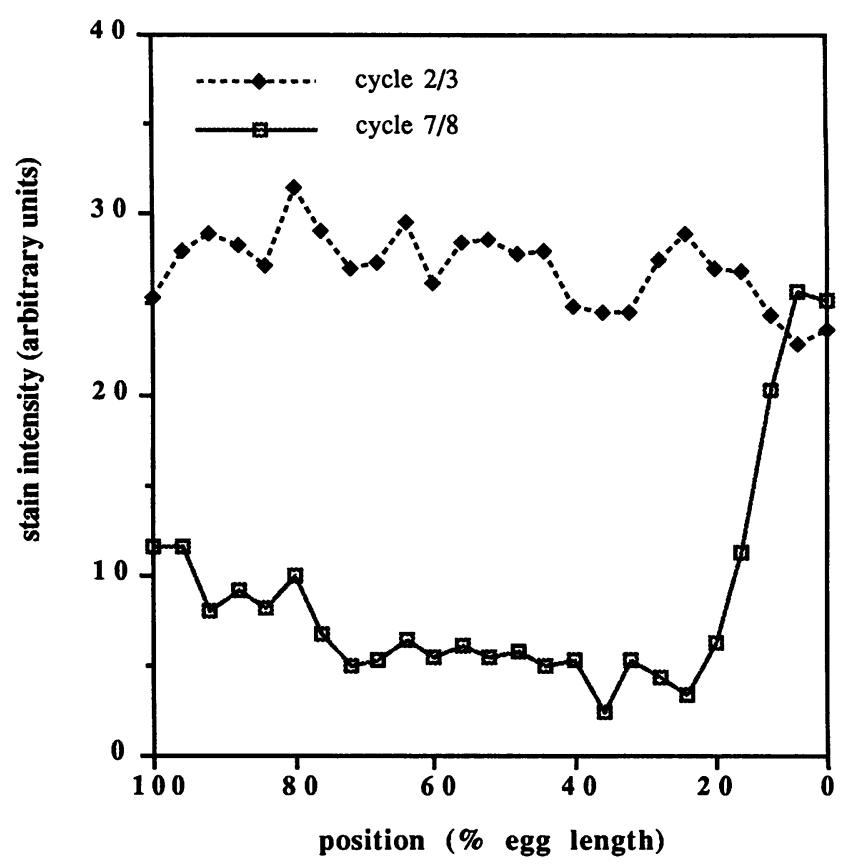

FIG. 2. Quantitative analysis of the distribution of Hsp83 RNA in wild-type embryos. The distribution of $H s p 83$ transcripts is shown at nuclear division cycle $2 / 3$ and at cycle $7 / 8$. While the amount of $H s p 83$ RNA at the posterior pole (0 to $5 \%$ egg length) remains constant, the $H s p 83$ RNA throughout the remainder of the embryo is degraded. Each point represents the mean value obtained from five embryos; standard deviations varied between 1.5 and 12.0 U. See Materials and Methods for details about the methods used in the production of these plots.

extension stages, in addition to the head and the pole cells, Hsp83 RNA can be detected in the neuroblasts (Fig. 1i). In mature embryos, high levels of $H s p 83$ RNA can be detected in the gonads (Fig. 1j) and in other tissues.

Maternally synthesized Hsp83 RNA is a component of the posterior polar plasm. We examined the distribution of Hsp83 RNA in embryos derived from females homozygous for maternal mutations affecting components of the posterior polar plasm. Maternal $H s p 83$ RNA is distributed normally in early-cleavage-stage embryos produced by females carrying mutations that affect the integrity of the posterior polar plasm, polar granules, and pole cell formation (cappuccino, spire, oskar, vasa, staufen, valois, and tudor) (examples are presented in Fig. 3a, c, and e). However, maternal Hsp83 RNA is no longer protected at the posterior poles of these embryos, and it is completely degraded between cycles 6 and 9, causing cellular blastoderm-stage embryos to exhibit only anterior, zygotic $H s p 83$ RNA (Fig. 3b, d, and f). In contrast, in mutants that are affected components of the polar plasm required for abdominal patterning but not for polar granule integrity or pole cell formation (nanos and pumilio), the posterior localization of $H s p 83$ RNA is normal (the nanos results are shown in Fig. $3 g$ and $h$ ). The loss of maternal Hsp83 RNA from the posterior of embryos lacking posterior polar plasm suggests that it is a component of the polar plasm and that it is protected from degradation by other components of the polar plasm, possibly the polar granules themselves.

Further evidence in support of this possibility comes from an analysis of the effects of mislocalization of the oskar RNA on the distribution pattern of maternal Hsp83 RNA. Mislo- 


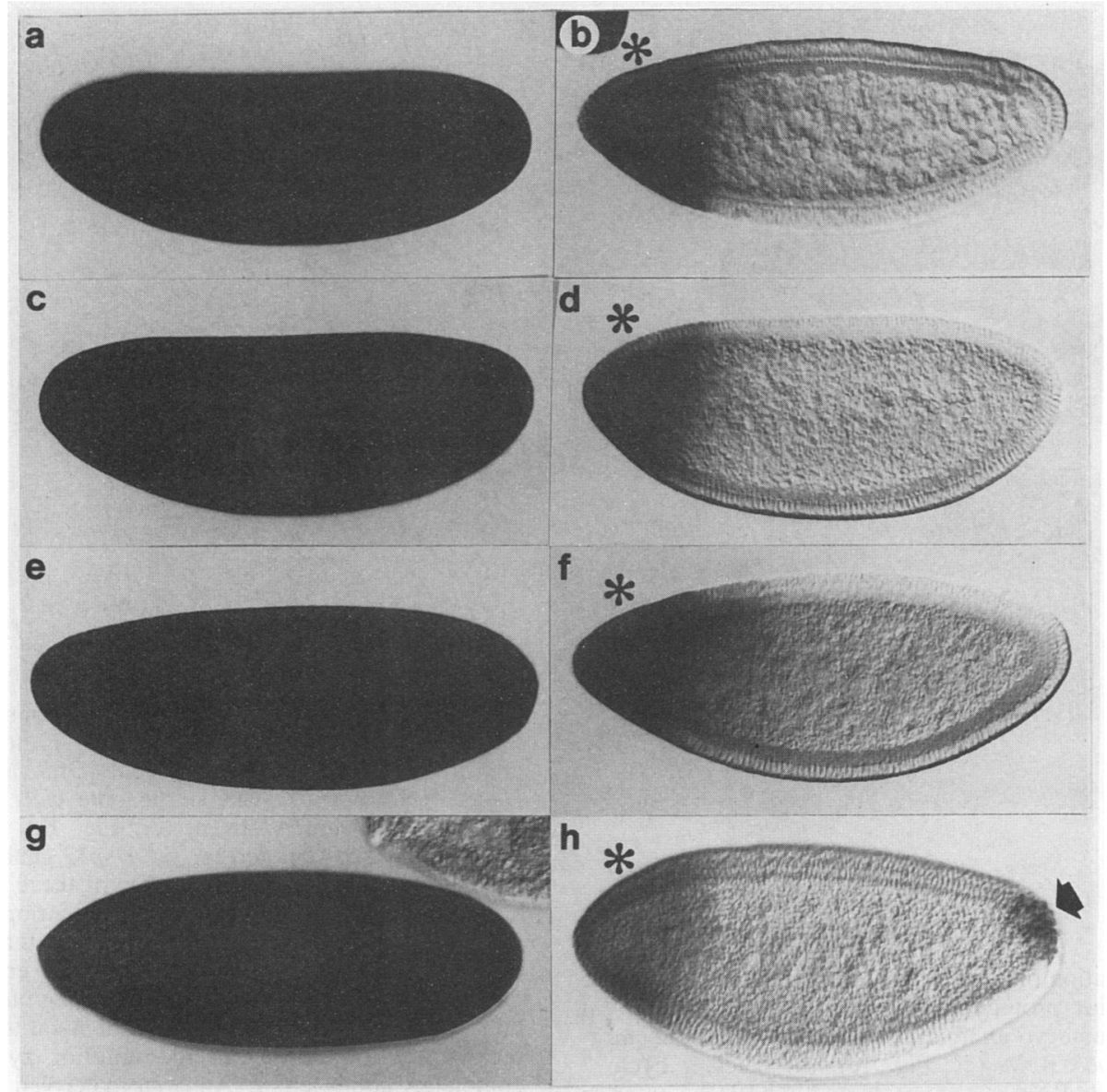

FIG. 3. Distribution of $H s p 83$ RNA in embryos produced by females homozygous for representative mutations that eliminate components of the posterior polar plasm and disrupt the integrity of the polar granules and the ability to form pole cells (a to f) or that eliminate components of the polar plasm without affecting the integrity of the polar granules or the ability to form pole cells ( $\mathrm{g}$ and $\mathrm{h}$ ). These are spire (a and $\mathrm{b}$ ), oskar (c and d), tudor (e and f), and nanos ( $\mathrm{g}$ and $\mathrm{h}$ ). Maternal Hsp83 RNA is distributed normally in the early-cleavage-stage embryos in all mutants (a, c, e, and $\mathrm{g}$ ). However, in mutants that eliminate polar granules, maternal Hsp83 RNA is not protected from degradation at the posterior pole and can no longer be detected by the cellular blastoderm stage (b, $d$, and $f$ ). In mutants in which the polar granules are present (e.g., nanos), protection of maternal $H s p 83$ RNA still occurs (arrow [h]). Note that, in all cases, the anterior zygotic expression of $H s p 83$ is normal at this stage (asterisks). The apparent lower level of anterior RNA in nanos (h) is a consequence of slight underdevelopment of the in situ staining reaction. In addition to the anterior expression, zygotic $H s p 83$ transcripts can be detected at 15 to $25 \%$ egg length in the embryos produced by cappuccino mothers (data not shown). For all embryos, anterior is to the left and dorsal is up.

calization of oskar RNA to the anterior pole of the early embryo via the bicoid ' 3 ' untranslated region (UTR) has been shown to result in the induction of germ cells anteriorly, a process dependent on the vasa and tudor gene products (15). We examined the dynamics of maternal Hsp83 RNA degradation and protection in oskar-bicoid $3^{\prime} U T R$ embryos. Maternal Hsp83 RNA is uniformly distributed in early oskarbicoid 3'UTR embryos (Fig. 4a); however, it is protected from degradation at both poles between nuclear cycles 6 and 8 (Fig. 4b). While protection of maternal $H s p 83$ RNA is now bipolar, the spatial pattern of anterior-protected $H s p 83$ RNA resembles that of bicoid RNA (Fig. 4b), a result consistent with the fact that ectopic anterior oskar RNA is distributed in a bicoid-like pattern (15). As at the posterior pole, this maternal Hsp83 RNA is taken up into the ectopic anterior pole cells; however, a significantly higher concentration of Hsp83 RNA remains in the anterior somatic cells underlying the ectopic pole cells than in the posterior somatic cells (Fig. $4 c$ and $d)$.

Spatially restricted zygotic expression of $\mathrm{Hsp} 83$ in the ante- rior of the embryo is controlled by bicoid. The earliest zygotic expression of $\mathrm{Hsp} 83$ is detected in the anterior third of the embryo during the late syncytial blastoderm stage, and this spatially restricted expression persists through the cellular blastoderm stage (Fig. 1h) and gastrulation (Fig. 1i). We have confirmed that this anterior expression is zygotic by demonstrating that it is absent in embryos homozygous for a deletion that removes the Hsp83 locus (Fig. 5a). The anterior determinant, bicoid, a homeodomain protein present in the early embryo in an anterior to posterior gradient $(12,13)$, regulates this anterior expression of $H s p 83$. In embryos that are derived from homozygous bicoid females, anterior Hsp83 expression is completely abolished while posterior localization of the maternally transcribed $H s p 83$ RNA is unaffected (Fig. 5b). This suggests that the Hsp83 gene is transcriptionally activated in response to bicoid. Zygotic expression of $\mathrm{Hsp} 83$ is not activated at the anterior of embryos that have oskar RNA mislocalized anteriorly (Fig. 4c), consistent with the expected absence of bicoid protein from these embryos (15). 


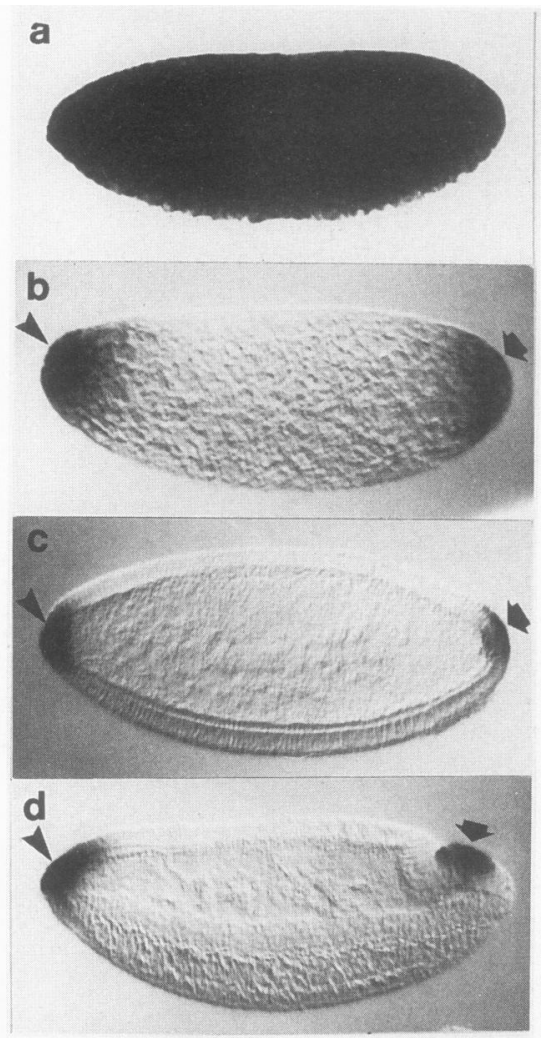

FIG. 4. Mislocalization of oskar RNA to the anterior embryonic pole results in anterior protection of maternal Hsp83 RNA. (a) Early-cleavage-stage embryo exhibiting a high, uniform concentration of maternal Hsp83 RNA (cf. Fig. 1e). (b) Nuclear cycle 7 embryo in which Hsp83 RNA is now protected at both the anterior (arrowhead) and the posterior (arrow) poles (cf. Fig. 1f). Note that the anterior protection pattern differs from that at the posterior pole and resembles that of bicoid RNA; this is because the mislocalized oskar-bicoid 3'UTR RNA is localized in a bicoid-like pattern (15). (c) Cellular blastoderm-stage embryo showing pole cells containing Hsp83 RNA at both poles (cf. Fig. 1h). In addition, there is substantially more ectopically protected $H s p 83$ RNA in the underlying anterior somatic cells than in the posterior ones. Note that there is no zygotic expression of $H s p 83$ in the anterior of the embryo (cf. Fig. 1h). (d) Gastrulating embryo showing Hsp83 RNA in the normal and ectopic pole cells, as well as persistent $H s p 83$ RNA in the underlying anterior somatic cells. Orientation of the embryos is as in Fig. 1.

Several gene products have been shown to localize the bicoid RNA to the anterior pole of the oocyte and early embryo $(1,58)$. The most extreme delocalization of bicoid RNA is produced by exuperantia mutations; in embryos derived from exuperantia females, bicoid RNA and protein are present in a shallow gradient that extends along most of the embryonic anteroposterior axis $(1,13,58)$. In such embryos, there is a marked posterior shift in the boundary of Hsp83 expression (Fig. 5c), consistent with the conclusion that anterior zygotic Hsp83 expression is dependent on the bicoid protein.

bicoid is known to directly regulate zygotic expression of the hunchback gene in the anterior half of the embryo (13). We examined Hsp83 expression in homozygous hunchback embryos in order to determine whether the bicoid regulation of $H s p 83$ is indirect, via zygotically expressed hunchback. There was no detectable effect of hunchback mutations on

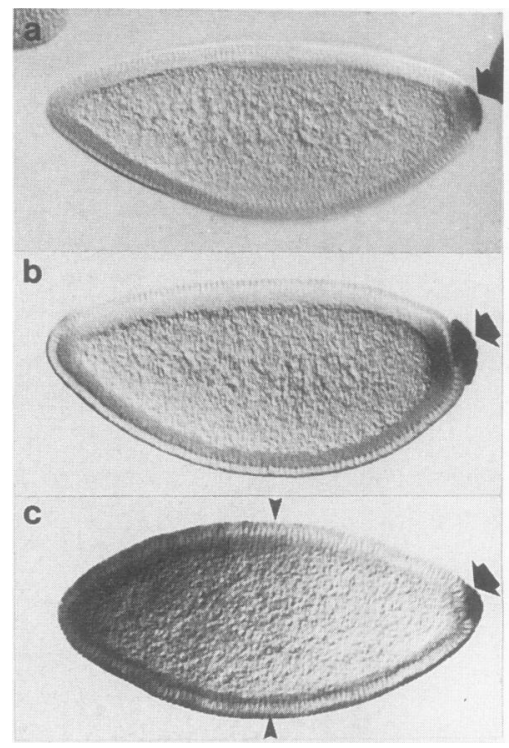

FIG. 5. Regulation of zygotic $H s p 83$ transcription by bicoid. (a) Cellular blastoderm-stage embryo that is homozygous for a deficiency that removes the $H s p 83$ gene [ $D f(3 L) H R 370]$. The maternal $H s p 83$ RNA can be seen in the pole cells (arrow), but anterior zygotic expression is absent (cf. Fig. 1h). The genetic cross to produce embryos deleted for the $H s p 83$ gene is given in Materials and Methods. Roughly one-quarter of these embryos exhibited no anterior expression of $H s p 83$, while posterior localization of maternal $H s p 83$ RNA was normal. We presume that these embryos were the ones lacking the Hsp83 gene. (b) An embryo derived from a female homozygous for bicoid ${ }^{E 1}$, a strong bicoid allele. The distribution of maternal Hsp83 RNA is identical to that of the wild type (arrow) (cf. Fig. 1h), but the anterior zygotic transcription is completely abolished. (c) An embryo derived from an exuperantia ${ }^{P J}$ female. The intensity of zygotic Hsp 83 expression is reduced and extends more posteriorly than in the wild type (to about $50 \%$ egg length; arrowheads) (cf. Fig. $1 \mathrm{~h}$ ). The distribution of maternal $H s p 83$ RNA is normal (arrow)

Hsp83 anterior expression (data not shown), suggesting that bicoid directly regulates $H s p 83$ expression. In addition, we have shown that mutations in torso, buttonhead, empty spiracles, orthodenticle, and giant, all of which function in programming head development and/or the development of the termini (for reviews, see references 7 and 16), are not required for activation of zygotic $H s p 83$ transcription in the anterior third of the embryo (data not shown).

Since there is normal temporal and spatial activation of a

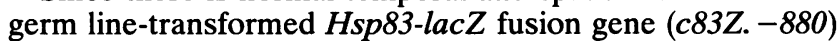
in the anterior third of the embryo (Fig. 6), cis-regulatory sequences sufficient for bicoid activation of zygotic Hsp83 transcription map to a $2.7-\mathrm{kb}$ region that includes $880 \mathrm{bp}$ of DNA $5^{\prime}$ to the Hsp83 transcription initiation site, its first exon, and its intron fused to the Escherichia coli lacZ gene (65). Detailed in vivo and in vitro analyses of sequences required for $H s p 83$ activation by bicoid are in progress and will be reported elsewhere (21a).

\section{DISCUSSION}

While it has been known for some time that the Hsp83 gene is expressed during normal development in the absence of heat shock $(33,65,66)$, we have shown that transcription of $H s p 83$ is dynamically regulated during oogenesis and embryogenesis. Maternal Hsp83 RNA is localized to the 


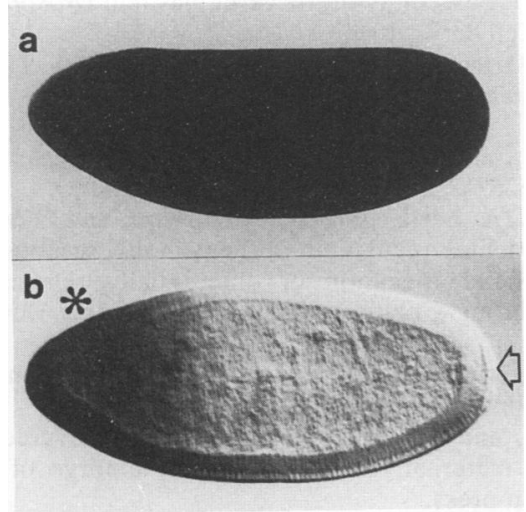

FIG. 6. Localization and expression of Hsp83-lacZ fusion transcripts. The embryos were derived from a female germ line transformed with $c 83 Z$. -880 (65), a construct that carries 880 bp of sequence $5^{\prime}$ to the Hsp 83 transcription start site, the first exon, and the intron, fused $300 \mathrm{bp}$ downstream of the translation start codon to the $E$. coli lacZ gene. The Hsp83-lacZ fusion transcripts were visualized with a $\beta$-galactosidase probe. (a) Early-cleavage-stage embryo. The fusion RNA is uniformly distributed at a high concentration throughout the embryo. (b) Syncytial blastoderm embryo, exhibiting fusion transcripts in the anterior (asterisk) but no posteriorly protected maternal RNA (unfilled arrow) (cf. Fig. 1g). Thus, the construct contains sequences sufficient for bicoid-dependent zygotic activation but lacks sequences that tag the Hsp83 RNA for posterior protection. Orientation is as in Fig. 1.

posterior pole of the early embryo by a novel mechanism involving a combination of generalized RNA degradation and local protection by components of the posterior polar plasm. Zygotic transcription of Hsp83 is restricted to the anterior third of the embryo and is absolutely dependent on bicoid, the anterior determinant. Here we discuss our results, their implications for understanding the mechanisms of RNA localization, and the possible developmental functions of $\mathrm{Hsp83.}$

A novel mechanism is used to localize maternal Hsp83 RNA to the posterior pole of the early embryo. It was hypothesized previously that RNAs could be localized in cells by a mechanism involving a combination of generalized degradation and local protection (19). However, to date there have been no examples of such a mechanism. Here we have shown that localization of maternally synthesized $H s p 83$ RNA to the posterior pole of the fertilized egg is accomplished by a combination of generalized degradation and local protection by components of the posterior polar plasm. High levels of $H s p 83$ RNA are distributed throughout the mature oocyte. An hour after fertilization, it is degraded rapidly throughout the embryo except at the posterior pole. Degradation of $H s p 83$ RNA occurs rapidly and uniformly in the anterior half of the embryo, but it is polarized in the posterior half of the embryo, resulting in production of a transient anteroposterior gradient of $H s p 83$ RNA. Posterior localization of maternal Hsp83 RNA thus represents the first example of a degradation-protection mechanism for RNA localization.

Posterior protection of maternal Hsp83 RNA is accomplished by components of the posterior polar plasm. We examined the effects on posterior protection of $H s p 83$ RNA of seven loss-of-function mutations that disrupt the posterior polar plasm, polar granules, and the ability to form germ cells. All of these result in degradation of Hsp83 RNA throughout the embryo without any posterior protection. Reciprocally, ectopic anterior localization of oskar RNA, which results in anterior assembly of components of the posterior polar plasm and formation of germ cells at the anterior pole (15), results in ectopic anterior protection of maternal Hsp83 RNA and its uptake into the ectopic germ cells. nanos and pumilio, which disrupt components of the posterior polar plasm required for abdominal development but not for germ cell formation, have no effect on posterior protection of $H s p 83$ RNA. Taken together, these results show that protection of Hsp83 RNA is accomplished by components of the posterior polar plasm that function in polar granule formation and in germ cell specification. It remains to be determined what gene products are involved in the degradation of the Hsp83 RNA throughout the remainder of the embryo.

Hsp83 and the germ line. Not only is Hsp83 RNA a component of the posterior polar plasm, but it is present in the pole cells and their germ line progeny throughout embryogenesis (this study) and larval development $(65,66)$ and in the adult (with two exceptions [see below]) $(65,66$; this study). Interestingly, the mouse $H s p 86$ gene, a close relative of Drosophila Hsp83 $(25,52)$, is expressed in germ cells (8, 20,38 ) as might be a human Hsp85 gene (34), suggesting that members of the $H s p 90$ gene family might serve similar functions in germ cells of diverse animal species (33).

Hsp83 RNA is absent from the Drosophila germ line in stage 1 germaria and is absent from the oocyte in stage 6 through $10 \mathrm{~A}$ egg chambers. We have yet to determine the functional significance of the cessation of $H s p 83$ transcription during these two periods; however, they correspond to the stages during which two key cell fate decisions are made. First, it has been shown that, by early germarial stage $2 \mathrm{~A}$, the pro-oocyte is already different from the pro-nurse cells $(31,60)$, suggesting that the choice between oocyte and nurse cell fate is initiated in region 1 of the germarium (31, $60)$. Second, communication between the oocyte and the surrounding follicle cells to specify the major aspects of its anteroposterior and dorsoventral axes occurs during stages 6 through $8(51)$. If $H s p 83$ protein is absent from the germ line during these stages, our results would suggest that $H s p 83$ must be eliminated in order to enable these cell fate decisions to occur. Despite directed attempts to obtain mutations in the Hsp83 gene, none have yet been identified (64). The production of loss-of-function phenotypes either by mutational or by molecular methods (e.g., antisense inactivation and dominant negative expression) will be required to further define the role of the $H s p 83$ gene in the germ line as well as in other tissues.

Zygotic $\mathrm{Hsp} 83$ transcription is regulated by the anterior determinant, bicoid. We have shown that zygotic activation of the Hsp83 gene occurs in the anterior third of the early embryo and is absolutely dependent on bicoid, the anterior determinant. Absence of functional bicoid protein eliminates zygotic activation of $\mathrm{Hsp} 83$ in the anterior third of the embryo. In contrast, extension of the bicoid protein distribution more posteriorly than in wild-type embryos results in an expanded domain of zygotic $H s p 83$ activation. None of an additional six genes that control anterior and/or terminal development (torso, hunchback, giant, empty spiracles, orthodenticle, and buttonhead) are required for anterior activation of $H s p 83$ transcription, suggesting that the $H s p 83$ gene might be activated directly by the bicoid homeodomain protein. Hsp83 is the first example of a gene encoding a cytoplasmically active regulatory protein that is spatially 
activated in response to the anterior genetic hierarchy Drosophila.

Functional significance of dynamic $H$ sp 83 expression during development. Proteins regulated by the mammalian Hsp 90 family of molecular chaperones include steroid hormone receptors, $s r c$ family tyrosine kinases, eIF- $2 \alpha$, protein kinase C, casein kinases, actin, and tubulin (for a review, see reference 44$)$. Given the dynamic spatial and temporal regulation of $H s p 83$ transcripts during Drosophila oogenesis and embryogenesis, it will be important in the future to determine whether $H s p 83$ protein expression is also dynamically controlled and whether it mirrors $H s p 83$ RNA. Should this be the case, it will then be important to identify the particular subset(s) of all possible partner proteins with which $H s p 83$ interacts in the germ cells and in the anterior cells of the embryo. Two potential partner proteins have been shown to be generally expressed throughout most of the early embryo $(29,32,62)$. One is the ecdysone receptor $(E c R)$, which is present throughout the embryo (32). If Hsp83 were to interact with this receptor in either the anterior cells or the pole cells, the complex spatial and temporal pattern of $H s p 83$ expression and localization could convert the generally expressed $E c R$ into a spatially and temporally regulated receptor. In addition, $D-\operatorname{src} 29 A$, a Drosophila src homolog, is expressed throughout the somatic blastoderm $(29,62)$, raising the possibility that $H s p 83$ regulates $\operatorname{src} 29 \mathrm{~A}$ activity in the anterior third of the embryo. Dynamic spatial and temporal regulation of $H s p 83$ might, thus, serve as a mechanism to confer spatially regulated activation and/or repression on more generally expressed partner proteins during Drosophila development.

\section{ACKNOWLEDGMENTS}

We thank P. Lasko, T. Schüpbach, A. Wohlwill, J. J. Bonner, H. Xiao, J. Lis, A. Ephrussi, R. Lehmann, and the Bowling Green and Indiana Drosophila Stock Centers for providing fly stocks; W. Fisher, J. Angus, and L. Richardson for technical assistance; E. Davidson for stimulating discussions about germ cell properties; and the following for critical comments on the manuscript: P. Sternberg, D. Weigel, K. Whittaker, and M. L. Yip.

D.D. was supported in part by graduate fellowships from the California Foundation for Biochemical Research, the Lucille P. Markey Charitable Trust, and the Howard Hughes Medical Institute; S.M.P. was supported by a Developmental Biology Grant from the Lucille P. Markey Charitable Trust (to H.D.L.) and an NIH Developmental Biology Postdoctoral Training Grant Fellowship; S.R.H. was supported by an NIH Predoctoral Training Grant and a Markey Charitable Trust Predoctoral Fellowship. This research was supported by a U.S. Public Health Service Program Project Grant (GM40499) and an award from the Searle Scholars Program of the Chicago Community Trust (both to H.D.L.).

\section{REFERENCES}

1. Berleth, T., M. Burri, G. Thoma, D. Bopp, S. Richstein, G. Frigerio, M. Noll, and C. Nüsslein-Volhard. 1988. The role of localization of bicoid RNA in organizing the anterior pattern of the Drosophila embryo. EMBO J. 7:1749-1756.

2. Blackman, R. K., and M. Meselson. 1986. Interspecific nucleotide sequence comparisons used to identify regulatory and structural features of the Drosophila hsp82 gene. J. Mol. Biol. 188:499-515.

3. Borkovich, K. A., F. W. Farrelly, D. B. Finkelstein, J. Taulien, and S. Lindquist. 1989. Hsp82 is an essential protein that is required in higher concentrations for growth of cells at higher temperatures. Mol. Cell. Biol. 9:3919-3930.

4. Bossy, B., L. M. C. Hall, and P. Spierer. 1984. Genetic activity along $315 \mathrm{~kb}$ of the Drosophila chromosome. EMBO J. 3:25372541 .

5. Boswell, R. E., and A. P. Mahowald. 1985. tudor, a gene required for assembly of the germ plasm in Drosophila melanogaster. Cell 43:97-104.

6. Campos-Ortega, J. A., and V. Hartenstein. 1985. The embryonic development of Drosophila melanogaster. Springer-Verlag, Berlin.

7. Cohen, S., and G. Jürgens. 1991. Drosophila headlines. Trends Genet. 7:267-272.

8. Curci, A., A. Bevilacqua, M. T. Fiorenza, and F. Mangia. 1991. Developmental regulation of heat-shock response in mouse oogenesis: identification of differentially responsive oocyte classes during Graafian follicle development. Dev. Biol. 144: 362-368.

9. Ding, D., and H. D. Lipshitz. Localized RNAs and their functions. Submitted for publication.

10. Ding, D., and H. D. Lipshitz. A molecular screen for polarlocalized maternal RNAs in the early embryo of Drosophila. Zygote, in press.

11. Ding, D., S. M. Parkhurst, and H. D. Lipshitz. 1993. Different genetic requirements for anterior RNA localization revealed by the distribution of Adducin-like transcripts during Drosophila oogenesis. Proc. Natl. Acad. Sci. USA, 90:2512-2516.

12. Driever, W., and C. Nüsslein-Volhard. 1988. The bicoid protein determines position in the Drosophila embryo in a concentration-dependent manner. Cell 54:95-104.

13. Driever, W., and C. Nüsslein-Volhard. 1988. A gradient of bicoid protein in Drosophila embryos. Cell 54:83-93.

14. Ephrussi, A., L. K. Dickinson, and R. Lehmann. 1991. oskar organizes the germ plasm and directs localization of the posterior determinant nanos. Cell 66:37-50.

15. Ephrussi, A., and R. Lehmann. 1992. Induction of germ cell formation by oskar. Nature (London) 358:387-392.

16. Finkelstein, R., and N. Perrimon. 1991. The molecular genetics of head development in Drosophila melanogaster. Development 112:899-912.

17. Frohnhöfer, H. G., and C. Nüsslein-Volhard. 1986. Organization of anterior pattern in the Drosophila embryo by the maternal gene bicoid. Nature (London) 324:120-125.

18. Golumbeski, G. S., A. Bardsley, F. Tax, and R. E. Boswell. 1991. tudor, a posterior-group gene of Drosophila melanogaster, encodes a novel protein and an mRNA localized during midoogenesis. Genes Dev. 5:2060-2070.

19. Gottlieb, E. 1990. Messenger RNA transport and localization. Curr. Opin. Cell Biol. 2:1080-1086.

20. Gruppi, C. M., Z. F. Zakeri, and D. J. Wolgemuth. 1991. Stage and lineage-regulated expression of two $H s p 90$ transcripts during mouse germ cell differentiation and embryogenesis. Mol. Reprod. Dev. 28:209-217.

21. Hackett, R. W., and J. T. Lis. 1983. Localization of the hsp83 RNA within a 3292 nucleotide sequence from the 63B heat shock locus of $D$. melanogaster. Nucleic Acids Res. 11:70117030 .

21a.Halsell, S. R., and H. D. Lipshitz. Unpublished data.

22. Hay, B., L. Ackerman, S. Barbel, L. Y. Jan, and Y. N. Jan. 1988. Identification of a component of Drosophila polar granules. Development 103:625-640.

23. Hay, B., L. Y. Jan, and Y. N. Jan. 1988. A protein component of Drosophila polar granules is encoded by vasa and has extensive sequence similarity to ATP-dependent helicases. Cell 55:577-587.

24. Hay, B., L. Y. Jan, and Y. N. Jan. 1990. Localization of vasa, a component of Drosophila polar granules, in maternal effect mutants that alter embryonic anteroposterior polarity. Development 109:425-433.

25. Hoffiman, T., and B. Hovemann. 1988. Heat-shock proteins, Hsp84 and Hsp86, of mice and men: two related genes encode formerly identified tumour-specific transplantation antigens. Gene 74:491-501.

26. Holmgren, R., K. Livak, R. Morimoto, R. Freund, and M. Meselson. 1979. Studies of cloned sequences from four Drosophila heat shock loci. Cell 18:1359-1370.

27. Huettner, A. F. 1923. The origin of the germ cells in Drosophila melanogaster. J. Morphol. 37:385-423.

28. Jongens, T. A., B. Hay, L. Y. Jan, and Y. N. Jan. 1992. The 
germ cell-less gene product: a posteriorly localized component necessary for germ cell development in Drosophila. Cell 70:569584.

29. Katzen, A. L., T. Kornberg, and J. M. Bishop. 1990. Diverse expression of $d s r c 29 A$, a gene related to $s r c$, during the life cycle of Drosophila melanogaster. Development 110:1169-1183.

30. Kim-Ha, J., J. L. Smith, and P. M. Macdonald. 1991. oskar mRNA is localized to the posterior pole of the Drosophila oocyte. Cell 66:23-35.

31. King, R. C. 1970. Ovarian development in Drosophila melanogaster. Academic Press, Inc., New York.

32. Koelle, M. R., W. S. Talbot, W. A. Segraves, M. T. Bender, P. Cherbas, and D. S. Hogness. 1991. The Drosophila EcR gene encodes an ecdysone receptor, a new member of the steroid receptor superfamily. Cell 67:1371-1389.

33. Kurtz, S., J. Rossi, L. Petko, and S. Lindquist. 1986. An ancient developmental induction: heat shock proteins induced in sporulation and oogenesis. Science 231:1154-1156.

34. Lai, B.-T., N. W. Chin, A. E. Stanek, W. Keh, and K. W. Lanks. 1984. Quantitation and intracellular localization of the $85 \mathrm{~K}$ heat shock protein by using monoclonal and polyclonal antibodies. Mol. Cell. Biol. 4:2802-2810.

35. Lantz, V., L. Ambrosio, and P. Schedl. 1992. The Drosophila orb gene is predicted to encode sex-specific germline RNAbinding proteins and has localized transcripts in ovaries and early embryos. Development 115:75-88.

36. Lasko, P. F., and M. Ashburner. 1988. The product of the Drosophila gene vasa is very similar to eukaryotic initiation factor 4A. Nature (London) 335:611-616.

37. Lasko, P. F., and M. Ashburner. 1990. Posterior localization of vasa protein correlates with, but is not sufficient for, pole cell development. Genes Dev. 4:905-921.

38. Lee, S.-J. 1990 . Expression of HSP86 in male germ cells. Mol. Cell. Biol. 10:3239-3242.

39. Lehmann, R., and C. Nüsslein-Volhard. 1986. Abdominal segmentation, pole cell formation and embryonic polarity require the localised activity of oskar, a maternal gene in Drosophila. Cell 47:141-152.

40. Lehmann, R., and C. Nüsslein-Volhard. 1987. hunchback, a gene required for segmentation of an anterior and posterior region of the Drosophila embryo. Dev. Biol. 119:402-417.

41. Lehmann, R., and C. Nüsslein-Volhard. 1987. Involvement of the pumilio gene in the transport of an abdominal signal in the Drosophila embryo. Nature (London) 329:167-170.

42. Lehmann, R., and C. Nüsslein-Volhard. 1991. The maternal gene nanos has a central role in posterior pattern formation of the Drosophila embryo. Development 112:679-693.

43. Lehner, C. F., and P. H. O'Farrell. 1990. The roles of Drosophila cyclins $A$ and $B$ in mitotic control. Cell 61:535-547.

44. Lindquist, S., and E. Craig. 1988. The heat-shock proteins. Annu. Rev. Genet. 22:631-677.

45. Macdonald, P. M. 1992. The Drosophila pumilio gene: an unusually long transcription unit and an unusual protein. Development 114:221-232.

46. Mahowald, A. P. 1962. Fine structure of pole cells and polar granules in Drosophila melanogaster. J. Exp. Zool. 151:201215.

47. Mahowald, A. P. 1968. Polar granules of Drosophila. II. Ultrastructural changes during early embryogenesis. J. Exp. Zool. 167:237-262.

48. Mahowald, A. P. 1971. Polar granules of Drosophila. III. The continuity of polar granules during the life cycle of Drosophila.
J. Exp. Zool. 176:329-349.

49. Mahowald, A. P. 1971. Polar granules of Drosophila. IV. Cytochemical studies showing loss of RNA from polar granules during early stages of embryogenesis. J. Exp. Zool. 176:345-352.

50. Manseau, L., and T. Schüpbach. 1989. cappuccino and spire: two unique maternal-effect loci required for both anteroposterior and dorsoventral patterns of the Drosophila embryo. Genes Dev. 3:1437-1452.

51. Manseau, L., and T. Schüpbach. 1989. The egg came first, of course! Trends Genet. 5:400-405.

52. Moore, S. K., C. Kozak, E. A. Robinson, S. J. Ullrich, and E. A. Apella. 1989. Murine 86- and 84-kDa heat shock proteins, cDNA sequences, chromosome assignments, and evolutionary origins. J. Biol. Chem. 264:5343-5351.

53. Palazzolo, M. J., B. A. Hamilton, D. Ding, C. H. Martin, D. A. Mead, R. C. Mierendorf, K. Vijay Raghavan, E. M. Meyerowitz, and H. D. Lipshitz. 1990. Phage lambda cDNA cloning vectors for subtractive hybridization, fusion protein expression and Cre-loxP automatic plasmid subcloning. Gene 88:25-36.

54. Raff, J. W., W. G. F. Whitfield, and D. M. Glover. 1990. Two distinct mechanisms localize cyclin $B$ transcripts in syncytial Drosophila embryos. Development 110:1249-1261.

55. Schüpbach, T., and E. Wieschaus. 1986. Maternal-effect mutations altering the anterior-posterior pattern of the Drosophila embryo. Roux's Arch. Dev. Biol. 195:302-317.

56. Schüpbach, T., and E. Wieschaus. 1989. Female sterile mutations on the second chromosome of Drosophila melanogaster. I. Maternal effect mutations. Genetics 121:101-117.

57. St. Johnston, D., D. Beuchle, and C. Nüsslein-Volhard. 1991. staufen, a gene required to localize maternal RNAs in the Drosophila egg. Cell 66:51-63.

58. St. Johnston, D., W. Driever, T. Berleth, S. Richstein, and C. Nüsslein-Volhard. 1989. Multiple steps in the localization of bicoid RNA to the anterior pole of the Drosophila oocyte. Development 107(Suppl.):13-19.

59. Struhl, G., K. Struhl, and P. M. Macdonald. 1989. The gradient morphogen bicoid is a concentration-dependent transcriptional activator. Cell 57:1259-1273.

60. Suter, B., L. M. Romberg, and R. Steward. 1989. Bicaudal-D, a Drosophila gene involved in developmental asymmetry: localized transcript accumulation in ovaries and sequence similarity to myosin heavy chain tail domains. Genes Dev. 3:1957-1968.

61. Tautz, D., and C. Pfeifle. 1989. A non-radioactive in situ hybridization method for the localization of specific RNAs in Drosophila embryos reveals translational control of the segmentation gene hunchback. Chromosoma 98:81-85.

62. Vincent, W. S., R. J. Gregory, and S. C. Wadsworth. 1989. Embryonic expression of a Drosophila src gene: alternate forms of the protein are expressed in segmental stripes and in the nervous system. Genes Dev. 3:334-347.

63. Wang, C., and R. Lehmann. 1991. Nanos is the localized posterior determinant in Drosophila. Cell 66:637-647.

64. Wohlwill, A. D., and J. J. Bonner. 1991. Genetic analysis of chromosome region 63 of Drosophila melanogaster. Genetics 128:763-775.

65. Xiao, H., and J. T. Lis. 1989. Heat shock and developmental regulation of the Drosophila melanogaster Hsp83 gene. Mol. Cell. Biol. 9:1746-1753.

66. Zimmerman, J. L., W. Petri, and M. Meselson. 1983. Accumulation of a specific subset of $D$. melanogaster heat shock mRNAs in normal development without heat shock. Cell 32: 1161-1170. 